\title{
Functions of O-fucosyltransferase in Notch trafficking and signaling: towards the end of a controversy? Nicolas Vodovar* and François Schweisguth*
}

Address: Ecole Normale Supérieure, CNRS UMR8542, 46 rue d'Ulm, 75005 Paris, France. *Present address: Institut Pasteur, CNRS URA2578, 25 rue du Dr Roux, 75015 Paris, France.

Correspondence: François Schweisguth. Email: schweisg@biologie.ens.fr

Published: 28 February 2008

Journal of Biology 2008, 7:7 (doi:10.1186/jbiol68)

The electronic version of this article is the complete one and can be found online at http://jbiol.com/content/7/2/7

(c) 2008 BioMed Central Ltd

\begin{abstract}
The precise role of the O-fucosyltransferase Ofutl in Notch-receptor trafficking has remained controversial. A recent study sheds new light on the non-catalytic activity of Ofut I and provides further evidence that Ofutl acts as a chaperone in the endoplasmic reticulum.
\end{abstract}

Notch proteins are evolutionarily conserved cell-surface receptors for transmembrane ligands of the DSL family (named after the Delta and Serrate ligands of Drosophila) $[1,2]$. Signaling by Notch regulates a broad range of cell-fate decisions during development and various human diseases, including cancers, have been associated with defects in Notch signaling.

The extracellular part of Notch contains 36 epidermal growth factor-like (EGF) repeats carrying the ligand-binding region and three Lin12/Notch repeats (LNRs) that limit proteolytic cleavage of the receptor at the S2 site, and hence limit its activation [2]. The EGF repeats are modified by two types of $O$-linked glycosylation, $\mathrm{O}$-glucosylation and $\mathrm{O}$-fucosylation (Figure 1a); both are important for Notch activity [3-6] (Figure 1a).

O-fucosylation of Notch is catalyzed by an O-fucosyltransferase (Pofut1 in mammals and Ofut 1 in Drosophila) $[7,8]$ that uses GDP-fucose as a substrate. The O-fucose residue added by this $O$-fucosyltransferase can be further elongated by the addition of an $N$-acetylglucosamine by Fringe, an EGF-O-fucose $\beta 1,3 \mathrm{~N}$-acetylglucosamyltransferase.
In Drosophila, the activity of Fringe is required for Notch signaling events involved in boundary formation, in which it reduces the affinity of Notch for Serrate while enhancing that for Delta $[9,10]$. Given that Ofut1 is thought to be the sole enzyme that $O$-fucosylates Notch, the activity of Fringe is predicted to require the $O$-fucosylation activity of Ofut 1 . However, Ofut 1 has additional functions, and other catalytic and non-catalytic activities have been proposed [8,11-16]. These include roles in the folding of Notch in the endoplasmic reticulum (ER), in Notch-ligand binding and in endocytic trafficking of Notch. These different activities have been incorporated into two distinct models (Figure 1b). There are several observations that support each of these models, and there has been controversy about which one is correct. A recent paper in BMC Biology by Kenneth Irvine and colleagues (Okajima et al. [17]) provides compelling evidence in favor of one of these models.

\section{The models and the supporting evidence}

The first model [16] proposes that Ofut1 acts in the ER, where it performs two separable functions: to O-fucosylate Notch, thereby modulating Notch-ligand interaction, and to 
(a)

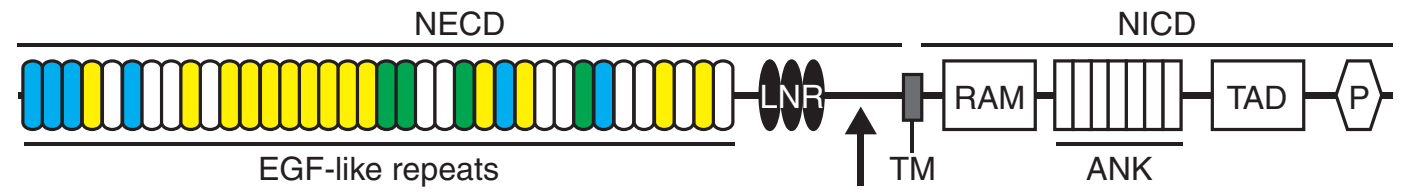

(b)

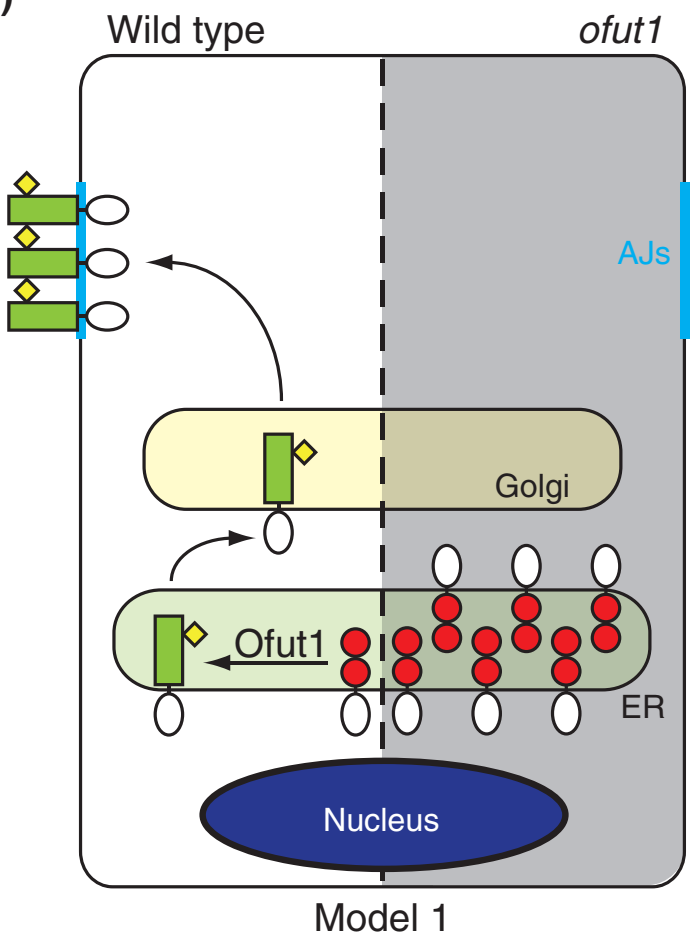

(c)

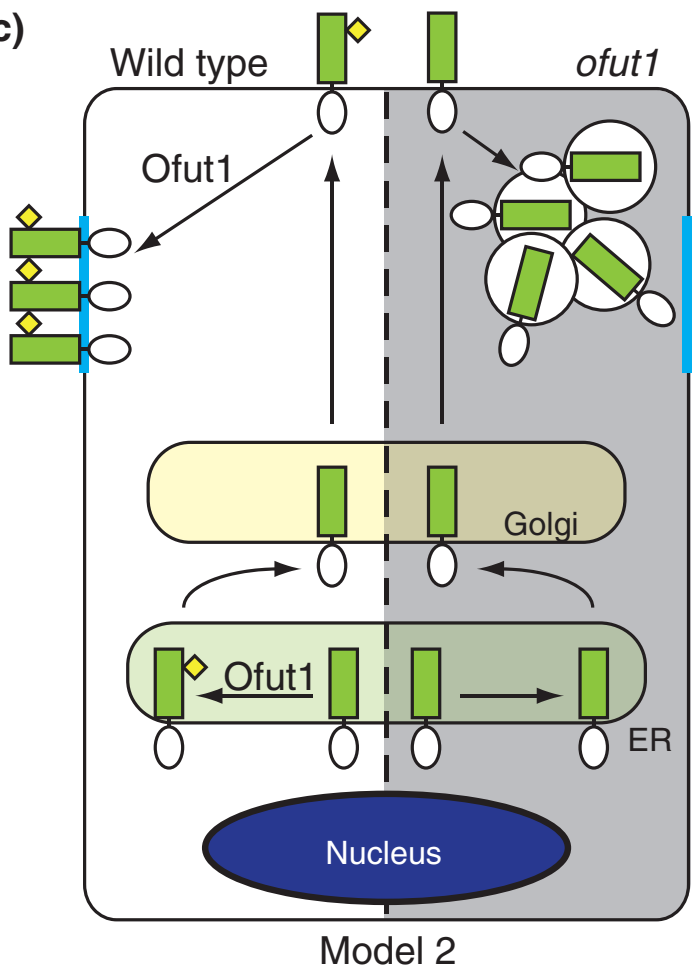

Figure I

O-fucosylation of Notch and functions of Ofut I. (a) Schematic representation of the Drosophila Notch receptor. The extracellular domain (NECD) is composed of three Lin I2/Notch repeats (LNRs) (black) and 36 EGF-like repeats; the EGF repeats are predicted to be either Oglucosylated (cyan), O-fucosylated (yellow) or doubly modified (green) adapted from [19]. The S2 cleavage site is indicated by an arrow. The intracellular domain (NICD) contains various elements involved in transcriptional activation: a RAM domain, seven ankyrin repeats (ANK), a transactivation domain (TAD) and a PEST domain (P). TM, transmembrane domain. (b,c) Two models for the roles of Ofutl in Notch trafficking. Each model is illustrated in a schematic cell that is shown as wild type on the left and ofut l mutant on the right, gray-shaded side. (b) Model I: in the ER, newly synthesized, unfolded Notch (with the NECD in red and the NICD in white) becomes properly folded (NECD in green) and is Ofucosylated by Ofutl (yellow diamond). It is then transported to the plasma membrane and adherens junctions (AJs, cyan) through the Golgi. In the absence of Ofut I, Notch remains unfolded and is retained in the ER. (c) Model 2: O-fucosylated Notch is first transported via the Golgi to the apical membrane and then transported to AJs by a transcytosis mechanism. In ofut I mutant cells, Notch goes to the membrane, is internalized and accumulates in an uncharacterized endocytic compartment.

promote the correct folding of the extracellular domain of Notch (a non-catalytic function). The evidence in support of this model is as follows. First, Ofut1 is a soluble ER protein $[16,18]$. It partially co-localizes with ER markers and acts, at least in part, in the ER: the carboxy-terminal extremity of Ofut 1 contains a Lys-Asp-Glu-Leu (KDEL)-like motif that is dispensable for its catalytic activity but is required for both ER retention and function $[16,18]$. Second, Ofut 1 is required for secretion of Notch [16]. In ofut1 mutant cells, the Notch protein accumulates in intracellular compartments marked by ER markers, and knockdown of Ofut1 using doublestranded RNA in cultured cells inhibits the secretion of a soluble version of the Notch extracellular domain (NECD) $[15,16]$. This activity of Ofut 1 does not require its $O-$ fucosylation activity because expression of a mutant version of Ofut 1 demonstrated to be catalytically dead (Ofut $1^{\text {R275A }}$ ) 
mostly rescues the Notch localization defects. In addition, Ofut 1 and Ofut $1^{\mathrm{R} 275 \mathrm{~A}}$ both bind the NECD, and expression of Ofut $1^{\mathrm{R} 275 \mathrm{~A}}$ in cultured cells can increase both the amount and the ligand-binding activity of secreted NECD [16]. Consistent with this proposed $O$-fucosylation-independent activity, Notch localizes at the cell cortex in GDP-mannose 4,6-dehydratase (Gmd) mutants [16], which contain no GDP-fucose [13], indicating that non-O-fucosylated Notch exits the ER.

Together, these results led to a simple model (model 1, Figure 1b) in which Ofut 1 acts as an ER chaperone to promote the proper folding of the EGF repeats of Notch, thereby ensuring its correct cell-surface localization and ligand-binding activity.

The second model $[12,13]$ proposes that Ofut 1 acts at two post-exocytosis steps in the trafficking of Notch: it regulates both the endocytosis of Notch [13] and its transcytosis from the apical plasma membrane to the adherens junctions (AJs) [12]. This model is supported by the following observations. First, Ofut1 is required for the proper distribution of Notch at AJs. In ofut1 mutant cells, Notch does not accumulate at AJs but instead accumulates into intracellular 'dots' that co-localize with ER markers only poorly [13]. In addition, surface-staining experiments indicate that a low level of Notch is also present at the surface of ofut 1 mutant cells [12]. These data were interpreted $[12,13]$ to suggest that Ofut 1 is not required for the ER exit and cell-surface delivery of Notch.

Second, Ofut 1 is required to regulate the endocytic trafficking of Notch, as monitored by antibody uptake. Anti-Notch antibodies are internalized in ofut 1 mutant cells, albeit at a lower level than in wild-type cells, but fail to accumulate in endosomes [13]. It is therefore proposed that Notch accumulates in an uncharacterized endocytic compartment in ofut 1 mutant cells. Finally, the second model is supported by the observation that a lower level of intracellular Notch is detected in Gmd mutant cells than in ofut1 single-mutant or ofut1, Gmd double-mutant cells $[12,13]$. This suggests that the endocytic activity of Ofut 1 does not depend on its catalytic activity. In addition, the failure of Notch to localize at AJs in Gmd mutant cells is interpreted in the light of a speculative model of the transcytosis of Notch to suggest that Ofut 1 acts in a catalysis-dependent manner to regulate the transcytosis of Notch [12]. We feel that this transcytosis model needs further experimental validation, so we will not detail the possible role of Ofut1 in this process further. We note, however, that this proposed role of Ofut1 in the transcytosis of Notch suggests that the $O$-fucosylation activity of Ofut 1 is required for Fringe-independent signaling events.
Together, these observations suggest a model (model 2, Figure 1c) in which Ofut 1 regulates the endocytic trafficking of Notch at a post-internalization step so that Notch accumulates in an undefined endocytic compartment in the absence of ofut1 activity.

Thus, these two models offer two different interpretations of the cellular basis of the ofut1 mutant phenotype: in model 1 , the reason Notch does not signal is because it is trapped in the ER, whereas in model 2, it is because Notch accumulates in an undefined endocytic compartment with a low level of (inactive) Notch at the surface.

One implication of model 2 is that a fraction of Ofut 1 must escape ER retention and act in the endocytic pathway. Consistent with this possibility, experiments using cultured cells indicate that a fraction of Ofut 1 is secreted, interacts with the extracellular part of Notch at the cell surface, and is internalized in a Notch-dependent manner [13]. In addition, the Notch localization defects seen in cells treated with ofut1 double-stranded RNA can be rescued by adding Ofut1containing conditioned medium [13]. Whether this happens in vivo is not clear. Indeed, ofut 1 acts in a cell-autonomous manner to regulate the localization of Notch, arguing that secretion is not important in this regulation [12]. Moreover, the observed rescue of the ofut 1 knockdown phenotype by extracellular Ofut 1 could also be consistent with model 1, given that endocytosed Ofut 1 may be transported back into the ER.

\section{Towards the end of a controversy}

So, how can we distinguish between the two models? Clearly, answering the following questions would help. Is Ofut 1 required in the ER or in an endocytic compartment? Does Notch reach the cell surface in the absence of Ofut1 activity? Where does Notch accumulate in ofut1 mutant cells? Is the O-fucosylation activity of Ofut 1 required for proper Notch localization and activity? Or is it only required for Fringe-dependent signaling? Okajima et al. [17] have addressed these issues and provide compelling evidence in favor of model 1.

Okajima et al. [17] have shown that the O-fucosylation activity of ofut 1 is required for Fringe-dependent but not for Fringe-independent signaling events. They found that embryos lacking both maternal and zygotic contributions of Gmd develop into larvae, as fringe mutant embryos do. The complete loss of ofut 1 activity results in a strong phenotype mimicking a loss of Notch activity that is rescued to larval viability by the expression of Ofut $1^{\mathrm{R} 275 \mathrm{~A}}$. These data show that the $O$-fucosylation activity of Ofut 1 is dispensable for Notch signaling in the embryo. This conclusion is entirely 
consistent with the observation that the activity of Fringe is dispensable in the embryo. It is therefore clear that nonfucosylated Notch can reach the cell surface and can signal.

Analysis by Okajima et al. [17] of the role of the Ofucosylation activity of ofut1 during wing imaginal disc development led to identical conclusions. Although the loss of ofut 1 activity in clones leads to phenotypes mimicking a loss of Notch activity, expression of Ofut $1^{\text {R275A }}$ rescued Notch receptor activity in these ofut 1 mutant cells and led to phenotypes mimicking a loss of fringe activity. This confirms that non-fucosylated Notch can signal, and further indicates that the fucosylation activity of ofut 1 is required only for Fringe-dependent signaling events. This therefore implies that the transcytosis step of model 2, which was proposed to be dependent on Ofut 1 catalytic activity, is not essential for Notch signaling.

Because the controversy over the exact role of Ofut 1 is in part due to methodological differences between the various studies and also to technical limitations in subcellular localization analysis, Okajima et al. [17] also re-examined the localization of Notch in ofut 1 mutant cells using a detergent-free cell-surface staining protocol. A striking difference in surface staining was observed between wildtype and ofut 1 mutant cells. This convincingly shows that Notch is not present at detectable levels at the surface of mutant cells. This contradicts the results obtained by Sasaki et al. [12], who used a different protocol to assay the presence of Notch at the cell surface of ofut 1 mutant cells. Whether the difference in protocols accounts for these opposite conclusions remains to be addressed experimentally. The second piece of evidence in support of a cell surface accumulation of Notch in ofut 1 mutant cells comes from antibody uptake experiments showing that anti-Notch antibodies can be internalized by ofut1 mutant cells [11]. However, as discussed by Okijama et al. [15], the low level of antibody uptake can probably be accounted for by the fluid-phase uptake of anti-Notch antibodies by live ofut1 mutant cells, followed by the specific retention of internalized antibodies by Notch accumulating intracellularly. Together, the published data are best interpreted as concluding that Notch does not reach the cell surface in ofut1 mutant cells.

Okajima et al. [17] next analyzed the distribution of Notch in ofut 1 mutant cells. Notch was shown to partially colocalize with four different ER markers that, in fact, show only partial co-localization among themselves. Thus, the only partially overlapping distribution of ER markers may explain the poor co-localization of Notch with the two ER markers seen by Sasamura et al. [13]. Okajima et al. [17] therefore propose that Notch accumulates in ofut 1 mutant cells in the ER, which is a heterogeneous organelle. Accordingly, Notch should co-localize better with the sum of the signals of the different ER markers. This remains to be tested.

In the light of these new data, it is clear that the O-fucosylation of Notch is primarily required for Fringe-dependent signaling events and that Ofut1 acts non-catalytically to regulate the exit of Notch from the ER. Thus, Ofut1 probably acts as a chaperone in the ER to promote the proper folding of the extracellular domain of Notch, as described in model 1 . Although the catalytic and non-catalytic activities of Ofut1 can be experimentally uncoupled, it is attractive to speculate that the O-fucosylation activity of Ofut1 participates in the quality-control mechanism that ensures that only properly folded Notch exits the ER. Further analysis of the trafficking of non-fucosylated Notch, produced for instance by Gmd mutant cells, would help address this issue.

\section{Acknowledgements}

We thank A. Bardin and C. Perdigoto for critical reading of the manuscript. N.V. is supported by the Agence Nationale pour la Recherche.

\section{References}

I. Schweisguth F: Notch signaling activity. Curr Biol 2004, I4:RI29RI38.

2. Bray SJ: Notch signalling: a simple pathway becomes complex. Nat Rev Mol Cell Biol 2006, 7:678-689.

3. Rampal R, Luther KB, Haltiwanger RS: Notch signaling in normal and disease states: possible therapies related to glycosylation. Curr Mol Med 2007, 7:427-445.

4. Stanley P: Regulation of Notch signaling by glycosylation. Curr Opin Struct Biol 2007, 17:530-535.

5. Acar M, Jafar-Nejad H, Takeuchi H, Rajan A, Ibrani D, Rana NA, Pan $\mathrm{H}$, Haltiwanger RS, Bellen HJ: Rumi is a CAPIO domain glycosyltransferase that modifies Notch and is required for Notch signaling. Cell 2008, 132:247-258.

6. Ge C, Stanley P: The O-fucose glycan in the ligand-binding domain of Notchl regulates embryogenesis and T cell development. Proc Natl Acad Sci USA 2008, 105:I539- 1544.

7. Wang Y, Shao L, Shi S, Harris RJ, Spellman MW, Stanley P, Haltiwanger RS: Modification of epidermal growth factor-like repeats with O-fucose. Molecular cloning and expression of a novel GDPfucose protein O-fucosyltransferase. J Biol Chem 200I, 276:4033840345.

8. Okajima T, Irvine KD: Regulation of notch signaling by O-linked fucose. Cell 2002, I I I:893-904.

9. Bruckner K, Perez L, Clausen H, Cohen S: Glycosyltransferase activity of Fringe modulates Notch-Delta interactions. Nature 2000, 406:4II-4I5.

10. Moloney DJ, Panin VM, Johnston SH, Chen J, Shao L, Wilson R, Wang Y, Stanley P, Irvine KD, Haltiwanger RS, Vogt TF: Fringe is a glycosyltransferase that modifies Notch. Nature 2000, 406:369375.

II. Shi S, Stanley P: Protein O-fucosyltransferase I is an essential component of Notch signaling pathways. Proc Natl Acad Sci USA 2003, 100:5234-5239.

12. Sasaki N, Sasamura T, Ishikawa HO, Kanai M, Ueda R, Saigo K, Matsuno K: Polarized exocytosis and transcytosis of Notch during its apical localization in Drosophila epithelial cells. Genes Cells 2007, 12:89-103. 
13. Sasamura T, Ishikawa HO, Sasaki N, Higashi S, Kanai M, Nakao S, Ayukawa T, Aigaki T, Noda K, Miyoshi E, Taniguchi N, Matsuno K: The O-fucosyltransferase O-futl is an extracellular component that is essential for the constitutive endocytic trafficking of Notch in Drosophila. Development 2007, 134: I347-I 356.

14. Sasamura T, Sasaki N, Miyashita F, Nakao S, Ishikawa HO, Ito M, Kitagawa M, Harigaya K, Spana E, Bilder D, Perrimon N, Matsuno $\mathrm{K}$ : neurotic, a novel maternal neurogenic gene, encodes an $O$ fucosyltransferase that is essential for Notch-Delta interactions. Development 2003, 130:4785-4795.

15. Okajima T, Xu A, Irvine KD: Modulation of notch-ligand binding by protein O-fucosyltransferase I and fringe. J Biol Chem 2003, 278:42340-42345

16. Okajima T, Xu A, Lei L, Irvine KD: Chaperone activity of protein O-fucosyltransferase I promotes notch receptor folding. Science 2005, 307:1599-1603.

17. Okajima T, Reddy B, Matsuda T, Irvine KD: Contributions of chaperone and glycosyltransferase activities of O-fucosyltransferase I to Notch signaling. BMC Biol 2008, 6:I.

18. Luo $Y$, Haltiwanger RS: O-fucosylation of notch occurs in the endoplasmic reticulum. J Biol Chem 2005, 280: I I289- I I 294.

19. Moloney DJ, Shair LH, Lu FM, Xia J, Locke R, Matta KL, Haltiwanger RS: Mammalian Notchl is modified with two unusual forms of O-linked glycosylation found on epidermal growth factorlike modules. J Biol Chem 2000, 275:9604-96II. 